\title{
EXCELLENT NORMAL LOCAL DOMAINS AND EXTENSIONS OF KRULL DOMAINS
}

\author{
WILLIAM HEINZER, CHRISTEL ROTTHAUS, AND SYLVIA WIEGAND
}

This paper is dedicated to Hans-Bjørn Foxby.

\begin{abstract}
We consider properties of extensions of Krull domains such as flatness that involve behavior of extensions and contractions of prime ideals. Let $(R, \mathbf{m})$ be an excellent normal local domain with field of fractions $K$, let $y$ be a nonzero element of $\mathbf{m}$ and let $R^{*}$ denote the $(y)$-adic completion of $R$. For elements $\tau_{1}, \ldots, \tau_{s}$ of $y R^{*}$ that are algebraically independent over $R$, we construct two associated Krull domains: an intersection domain $A:=$ $K\left(\tau_{1}, \ldots \tau_{s}\right) \cap R^{*}$ and its approximation domain $B$; see Setting 2.2

If in addition $R$ is countable with $\operatorname{dim} R \geq 2$, we prove that there exist elements $\tau_{1}, \ldots, \tau_{s}, \ldots$ as above such that, for each $s \in \mathbb{N}$, the extension $R\left[\tau_{1}, \ldots, \tau_{s}\right] \hookrightarrow R^{*}[1 / y]$ is flat; equivalently, $B=A$ and $A$ is Noetherian. Using this result we establish the existence of a normal Noetherian local domain $B$ such that: $B$ dominates $R ; B$ has $(y)$-adic completion $R^{*}$; and $B$ contains a height-one prime ideal $\mathbf{p}$ such that $R^{*} / \mathbf{p} R^{*}$ is not reduced. Thus $B$ is not a Nagata domain and hence is not excellent.

We present several theorems involving the construction. These theorems yield examples where $B \subsetneq A$ and $A$ is Noetherian while $B$ is not Noetherian; and other examples where $B=A$ and $A$ is not Noetherian.
\end{abstract}

\section{INTRODUCTION}

About twenty years ago Judy Sally gave an expository talk on the following question:

Question 1.1. What rings lie between a Noetherian integral domain $S$ and its field of fractions $\mathcal{Q}(S)$ ?

We are inspired by work of Shreeram Abhyankar such as that in his paper [1] to ask the following related question: 1

Question 1.2. Let $I$ be an ideal of a Noetherian integral domain $R$ and let $R^{*}$ denote the $I$-adic completion of $R$. What rings lie between $R$ and $R^{*}$ ?

1991 Mathematics Subject Classification. Primary 13B35, 13J10, 13A15.

Key words and phrases. power series, Noetherian and non-Noetherian integral domains.

The authors are grateful for the hospitality, cooperation and support of Michigan State, Nebraska, Purdue and the CIRM at Luminy, France, where several work sessions on related research were conducted. We thank the referee for a careful reading of the paper and helpful comments.

${ }^{1}$ Ram's work demonstrates the vastness of power series rings. The authors have fond memories of many pleasant conversations with him concerning power series. 
A wide variety of integral domains fit the descriptions of both Questions 1.1 and 1.2. Let $(R, \mathbf{m})$ be an excellent normal local domain and let $S$ be a polynomial ring in finitely many variables over $R$. In work over a number of years related to these questions, the authors have been developing techniques for constructing examples that are birational extensions of $S$ and also subrings of an ideal-adic completion of $R$. Classical constructions of Noetherian integral domains with interesting properties, such as failure to be a Nagata ring, have been given by Akizuki, Schmidt, Nagata and others, 2], 10, 7]. We recall that a ring $A$ is a Nagata ring if $A$ is Noetherian and if the integral closure of $A / P$ in $L$ is finite over $A / P$, for every prime ideal $P$ of $A$ and every field $L$ finite algebraic over the field of fractions of $A / P$, [6, page 264].

We often use in our construction the completion of an excellent normal local domain $(R, \mathbf{m})$ with respect to a principal ideal $y R$, where $y$ is a nonzero nonunit of $R$. The $(y)$-adic completion $R^{*}$ of $R$ may be regarded as either an inverse limit or as a homomorphic image of a formal power series ring $R[[z]]$ over $R$. Thus we have

$$
R^{*}=\lim _{n}\left(\frac{R}{y^{n} R}\right)=\frac{R[[z]]}{(z-y) R[[z]]}
$$

An element $\tau$ of $R^{*}$ has an expression as a power series in $y$ with coefficients in $R$ It is often the case that there exist elements $\tau_{1}, \ldots, \tau_{n}$ in $R^{*}$ that are algebraically independent over $R$. An elementary cardinality argument shows this is always the case if $R$ is countable. Assume that $\tau_{1}, \ldots, \tau_{n}$ in $R^{*}$ are algebraically independent over $R$. By modifying $\tau_{i}$ by an element in $R$, we may assume that each $\tau_{i} \in y R^{*}$. Let $S:=R\left[\tau_{1}, \ldots, \tau_{n}\right]$. Then $S$ is both a subring of $R^{*}$ and a polynomial ring in $n$ variables over $R$. Although the expression for the $\tau_{i}$ as power series in $y$ with coefficients in $R$ is not unique, we use it to construct an integral domain $B$ that is a directed union of localized polynomial rings over $R$.

The construction we consider associates with $R$ and $\tau_{1}, \ldots, \tau_{n}$ the following two integral domains:

(1) an intersection domain $A:=\mathcal{Q}(S) \cap R^{*}$, and

(2) an integral domain $B \subseteq A$ that approximates $A$.

The integral domain $B$ is a directed union of localized polynomial rings in $n$ variables over $R$. The rings $B$ and $A$ are birational extensions of the polynomial ring $S$ and subrings of $R^{*}$. Thus they fit the descriptions of both Questions 1.1 and 1.2 For integral domains $A$ and $B$ obtained as above, we ask:

Questions 1.3. For a given excellent normal local domain $(R, \mathbf{m})$ and elements $\tau_{1}, \ldots, \tau_{n}$ as above, what properties do the constructed rings $A$ and $B$ have, and what criteria determine these properties? 
Our work in this article concerning Questions 1.3 focuses primarily on the case where the base ring $R$ is an excellent normal local domain. The intersection domain $A=\mathcal{Q}(S) \cap R^{*}$ may fail to be Noetherian even though $R$, and therefore $R^{*}$, is an excellent normal local domain. However, the intersection domain $A$ is always a Krull domain, and the (y)-adic completion of $A$ is $R^{*}$. Thus, in order to present an iterative procedure, in Section 2 we present many of the properties we study with the following Krull domain setting:

Setting 1.4. Let $(T, \mathbf{n})$ be a local Krull domain with field of fractions $\mathcal{Q}(T)$. Assume that $y \in \mathbf{n}$ is a nonzero element such that the $(y)$-adic completion $\left(T^{*}, \mathbf{n}^{*}\right)$ of $T$ is an analytically normal Noetherian local domain. Since the $\mathbf{n}$-adic completion of $T$ is the same as the $\mathbf{n}^{*}$-adic completion of $T^{*}$, it follows that the $\mathbf{n}$-adic completion $\widehat{T}$ of $T$ is also a normal Noetherian local domain. Let $\mathcal{Q}\left(T^{*}\right)$ denote the field of fractions of $T^{*}$. Since $T^{*}$ is Noetherian, $\widehat{T}$ is faithfully flat over $T^{*}$ and we have $T^{*}=\widehat{T} \cap \mathcal{Q}\left(T^{*}\right)$. Therefore $\mathcal{Q}(T) \cap T^{*}=\mathcal{Q}(T) \cap \widehat{T}$. Assume that $T=\mathcal{Q}(T) \cap T^{*}$, and let $d$ denote the dimension of the Noetherian domain $T^{*}$. It follows that $d$ is also the dimension of $\widehat{T}$.

Let $\tau_{1} \ldots, \tau_{s}$ be elements of $y T^{*}$ that are algebraically independent over $T$. We consider the extensions

$$
T \hookrightarrow T\left[\tau_{1} \ldots, \tau_{s}\right] \hookrightarrow A:=\mathcal{Q}(T)\left(\tau_{1}, \ldots, \tau_{s}\right) \cap T^{*} \hookrightarrow T^{*} .
$$

In particular, the following map is critical:

$$
\varphi: T\left[\tau_{1} \ldots, \tau_{s}\right] \hookrightarrow T^{*}[1 / y] .
$$

The intersection ring $A=\mathcal{Q}(T)\left(\tau_{1}, \ldots, \tau_{s}\right) \cap T^{*}$ and its approximating ring $B$ may be Noetherian or not, or excellent or not. Examples 4.3 and 4.8 demonstrate that one may have $B=A$, or $B \subsetneq A$. Properties of the rings $B$ and $A$ are related to properties of the map $\varphi$ of Equation 1.4,0; this is illustrated by two conclusions of Theorem 2.6

(1) $B$ is Noetherian if and only if $\varphi$ is flat.

(2) If $B$ is Noetherian, then $B=A$.

In general, by Theorem 2.9, we have $B=A$ if and only if $\varphi$ satisfies the weak flatness property in Definitions 2.7 below. We describe in Section 2 the construction of $B$.

The rings $T\left[\tau_{1}, \ldots, \tau_{s}\right]$ and $T^{*}[1 / y]$ in Equation [1.4. 0 are Krull domains as are also the constructed rings $B \hookrightarrow A$. We demonstrate connections between properties of the extension of Krull domains defined by the map $\varphi$ in Equation 1.4.0, and properties of the Krull domains $B$ and $A$. Some of our results hold for Krull domains as in Setting 1.4 for others we restrict to the case where $T=R$ is an excellent normal local domain. 
It is useful to give a name for the elements $\tau_{1}, \cdots, \tau_{s}$ in case the map $\varphi$ of Equation 1.4. 0 is flat.

Definition 1.5. Assume Setting 1.4 thus $T$ is a local Krull domain and $y$ is a nonzero nonunit element of $T$ such that the $(y)$-adic completion $T^{*}$ of $T$ is an analytically normal Noetherian local domain with $T=\mathcal{Q}(T) \cap T^{*}$. Elements $\tau_{1} \ldots, \tau_{s} \in y T^{*}$ that are algebraically independent over $T$ are said to be primarily limit-intersecting in $y$ over $T$ provided the inclusion map

$$
\varphi: T\left[\tau_{1}, \ldots, \tau_{s}\right] \hookrightarrow T^{*}[1 / y]
$$

is flat. An infinite set $\left\{\tau_{i}\right\}_{i=1}^{\infty}$ of elements in $y T^{*}$ that are algebraically independent over $T$ is said to be primarily limit-intersecting in $y$ over $T$ if for each positive integer $s$, the elements $\tau_{1}, \ldots, \tau_{s}$ are primarily limit-intersecting in $y$ over $T$.

It is natural to ask about the existence of primarily limit-intersecting elements:

Question 1.6. Let $R$ be an excellent normal local domain with $\operatorname{dim} R=d \geq 2$, let $y$ be a nonzero element in the maximal ideal $\mathbf{m}$ of $R$, and let $R^{*}$ be the $(y)$-adic completion of $R$. Under what conditions on $R$ do there exist elements that are primarily limit-intersecting in $y$ over $R$ ?

With notation as in Question 1.6, we describe in Theorem 3.5 and Remark 3.7 necessary and sufficient conditions that an element $\tau \in y R^{*}$ be primarily limitintersecting in $y$ over $R$. If $R$ is countable, we prove in Theorem 3.12 the existence of an infinite sequence $\tau_{1}, \ldots, \tau_{s}, \ldots \in y R^{*}$ of elements that are primarily limitintersecting in $y$ over $R$. We show in Theorem 3.13 that in general for an element $\eta \in y R^{*}$ that is primarily limit-intersecting in $y$ over $R$, the constructed Noetherian domain

$$
B=A=R^{*} \cap \mathcal{Q}(R[\eta])
$$

may fail to be excellent.

In Section 4 we present two theorems involving the construction. These theorems yield examples where $B \subsetneq A$ and $A$ is Noetherian while $B$ is not Noetherian; and other examples where $B=A$ and $A$ is not Noetherian. We describe several examples obtained by iteration of the construction considered in Section 3 .

\section{BASIC PROPERTIES AND THE APPROXIMATION DOMAIN}

In this section we give background and terminology. We generally assume Setting 1.4 in this section. First we illustrate the construction of an intersection domain $A$ and develop the terminology necessary for an approximation domain $B$ in what we consider the easiest example that can be constructed. 
Example 2.1. The "easiest" example. Let $k$ be a field, for example, $k=\mathbb{Q}$, the rational numbers, and let $x$ be an indeterminate over $k$. Let $R=k[x]_{(x)}$ and $R^{*}=k[[x]]$, the power series ring in $x$ over $k$ and the $x$-adic completion of $R$. Let $\tau \in x k[[x]]$ be algebraically independent over $R$; for example, if $k=\mathbb{Q}$, we could take $\tau=e^{x}-1$. Define $A$, the intersection domain associated to $\tau$ over $R$ by

$$
A=k(x, \tau) \cap k[[x]] .
$$

In this case $A$ is a rank-one discrete valuation ring (DVR) because it is the intersection of the DVR $k[[x]]$ with a subfield of $k((x))$ that is not contained in $k$. Thus $A$ is a Noetherian one-dimensional regular local ring (RLR) and the unique maximal ideal is $x A$.

We apply approximation techniques to more precisely describe the elements that are in $A$. In order to define an approximation domain $B$ that goes with $A$, write

$$
\tau=\sum_{i=1}^{\infty} a_{i} x^{i}
$$

where the $a_{i} \in k$. Define $\tau_{0}=\tau$. For each $n \in \mathbb{N}$, define the $n^{\text {th }}$ endpiece of $\tau$, denoted $\tau_{n}$, and define rings $U_{n}$ and $B_{n}$ by

$$
\tau_{n}=\sum_{i=n+1}^{\infty} a_{i} x^{i-n}, \quad U_{n}=k\left[x, \tau_{n}\right] \quad \text { and } \quad B_{n}=k\left[x, \tau_{n}\right]_{\left(x, \tau_{n}\right)} .
$$

Set

$$
U=\cup_{n=0}^{\infty} U_{n} \quad \text { and } \quad B=\cup_{n=0}^{\infty} B_{n} .
$$

It is straightforward to show that $A=B$ in Example 2.1 see [4, Chapter 6]. Since the extension $k\left[x, \tau_{n}\right] \hookrightarrow A$ does not satisfy the dimension inequality [6, p. 119], the ring $A=B$ is not the localization of a finitely generated algebra over $k$.

In general the intersection of a normal Noetherian domain with a subfield of its field of fractions is a Krull domain, but need not be Noetherian. A directed union of normal Noetherian domains may be a non-Noetherian Krull domain. Thus, in order to be able to iterate our construction, we consider a local Krull domain $(T, \mathbf{n})$ that is not assumed to be Noetherian, but is assumed to have a Noetherian completion. To distinguish from the Noetherian hypothesis on $R$, we let $T$ denote the base domain.

\section{The construction of the approximation domain.}

Setting and Notation 2.2. Let $(T, \mathbf{n})$ be a local Krull domain with field of fractions $F$. Assume there exists a nonzero element $y \in \mathbf{n}$ such that the $y$-adic completion $(\widehat{T,(y)}):=\left(T^{*}, \mathbf{n}^{*}\right)$ of $T$ is an analytically normal Noetherian local domain. It then follows that the $\mathbf{n}$-adic completion $\widehat{T}$ of $T$ is also a normal Noetherian local domain, since the $\mathbf{n}$-adic completion of $T$ is the same as the $\mathbf{n}^{*}$-adic 
completion of $T^{*}$. Since $T^{*}$ is Noetherian, if $F^{*}$ denotes the field of fractions of $T^{*}$, then $T^{*}=\widehat{T} \cap F^{*}$. Therefore $F \cap T^{*}=F \cap \widehat{T}$. Let $d$ denote the dimension of the Noetherian domain $T^{*}$. It follows that $d$ is also the dimension of $\widehat{T}$. 2

(1) Assume that $T=F \cap T^{*}=F \cap \widehat{T}$, or equivalently by Proposition 2.8 1, that $T^{*}$ and $\widehat{T}$ are weakly flat over $T$.

(2) Let $\widehat{T}[1 / y]$ denote the localization of $\widehat{T}$ at the powers of $y$, and similarly, let $T^{*}[1 / y]$ denote the localization of $T^{*}$ at the powers of $y$. The domains $\widehat{T}[1 / y]$ and $T^{*}[1 / y]$ have dimension $d-1$.

(3) Let $\tau_{1}, \ldots, \tau_{s} \in \mathbf{n}^{*}$ be algebraically independent over $F$.

(4) For each $i$ with $1 \leq i \leq s$, we have an expansion $\tau_{i}:=\sum_{j=1}^{\infty} c_{i j} y^{j}$ where $c_{i j} \in T$.

(5) For each $n \in \mathbb{N}$ and each $i$ with $1 \leq i \leq s$, we define the $n^{\text {th }}$-endpiece $\tau_{\text {in }}$ of $\tau_{i}$ with respect to $y$ as in Example 2.1

$$
\tau_{i n}:=\Sigma_{j=n+1}^{\infty} c_{i j} y^{j-n} .
$$

Thus we have $\tau_{i n}=y \tau_{i, n+1}+c_{i, n+1} y$.

(6) For each $n \in \mathbb{N}$, we define $B_{n}:=T\left[\tau_{1 n}, \ldots, \tau_{s n}\right]_{\left(\mathbf{n}, \tau_{1 n}, \ldots, \tau_{s n}\right)}$. In view of (5), we have $B_{n} \subseteq B_{n+1}$ and $B_{n+1}$ dominates $B_{n}$ for each $n$. We define

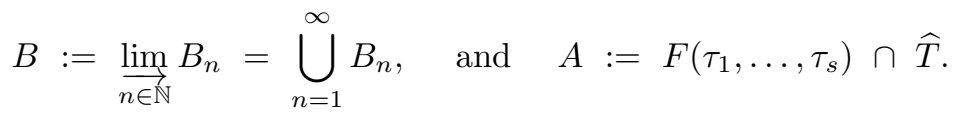

Thus, $B$ and $A$ are local Krull domains and $A$ birationally dominates $B$. We are especially interested in conditions which imply that $B=A$.

(7) Let $A^{*}$ denote the $y$-adic completion of $A$ and let $B^{*}$ denote the $y$-adic completion of $B$.

Remarks 2.3. The definitions of $B$ and $B_{n}$ are independent of representations for $\tau_{1}, \ldots, \tau_{s}$ as power series in $y$ with coefficients in $T$; see [4, Proposition 21.6].

\section{Properties of the construction.}

The following theorem is proved in 4, Theorem 21.7].

Theorem 2.4. Assume the setting and notation of (2.2). Then the intermediate rings $B_{n}, B$ and $A$ have the following properties:

(1) $y A=y T^{*} \cap A$ and $y B=y A \cap B=y T^{*} \cap B$. More generally, for every $t \in \mathbb{N}$, we have $y^{t} A=y^{t} T^{*} \cap A$ and $y^{t} B=y^{t} A \cap B=y^{t} T^{*} \cap B$.

(2) $T / y^{t} T=B / y^{t} B=A / y^{t} A=T^{*} / y^{t} T^{*}$, for each positive integer $t$.

\footnotetext{
${ }^{2}$ If $T$ is Noetherian, then $d$ is also the dimension of $T$. However, if $T$ is not Noetherian, then the dimension of $T$ may be greater than $d$. This is illustrated by taking $T$ to be the $\operatorname{ring} B$ of Example 4.3
} 
(3) Every ideal of T,B or A that contains $y$ is finitely generated by elements of $T$. In particular, the maximal ideal $\mathbf{n}$ of $T$ is finitely generated, and the maximal ideals of $B$ and $A$ are $\mathbf{n} B$ and $\mathbf{n} A$.

(4) For every $n \in \mathbb{N}: y B \cap B_{n}=\left(y, \tau_{1 n}, \ldots, \tau_{s n}\right) B_{n}$, an ideal of $B_{n}$ of height $s+1$.

(5) Let $P \in \operatorname{Spec}(A)$ be minimal over $y A$, and let $Q=P \cap B$ and $W=P \cap T$. Then $T_{W} \subseteq B_{Q}=A_{P}$, and all three localizations are DVRs.

(6) For every $n \in \mathbb{N}, B[1 / y]$ is a localization of $B_{n}$, i.e., for each $n \in \mathbb{N}$, there exists a multiplicatively closed subset $S_{n}$ of $B_{n}$ such that $B[1 / y]=S_{n}^{-1} B_{n}$.

(7) $B=B[1 / y] \cap B_{\mathbf{q}_{1}} \cap \cdots \cap B_{\mathbf{q}_{r}}$, where $\mathbf{q}_{1}, \ldots, \mathbf{q}_{r}$ are the prime ideals of $B$ minimal over $y B$.

The next theorem from [4, Theorem 21.8] is also useful in the sequel.

Theorem 2.5. With the setting and notation of (2.2), the intermediate rings $A$ and $B$ have the following properties:

(1) $A$ and $B$ are local Krull domains.

(2) $B \subseteq A$, with $A$ dominating $B$.

(3) $A^{*}=B^{*}=T^{*}$.

(4) If $B$ is Noetherian, then $B=A$.

Moreover, if $T$ is a unique factorization domain (UFD) and $y$ is a prime element of $T$, then $B$ is a UFD.

We use the following theorem [4, Theorem 21.13] to establish the Noetherian property.

Theorem 2.6. Assume the notation of Setting 2.2. Thus $(T, \mathbf{n})$ is a local Krull domain with field of fractions $F$, and $y \in \mathbf{n}$ is such that the (y)-adic completion $\left(T^{*}, \mathbf{n}^{*}\right)$ of $T$ is an analytically normal Noetherian local domain and $T=T^{*} \cap F$. For elements $\tau_{1}, \ldots, \tau_{s} \in \mathbf{n}^{*}$ that are algebraically independent over $T$, the following are equivalent:

(1) The extension $T\left[\tau_{1}, \ldots, \tau_{s}\right] \hookrightarrow T^{*}[1 / y]$ is flat.

(2) The elements $\tau_{1}, \ldots, \tau_{s}$ are primarily limit-intersecting in $y$ over $T$.

(3) The intermediate rings $A$ and $B$ are equal and are Noetherian.

(4) The constructed ring $B$ is Noetherian.

Moreover, if these equivalent conditions hold, then the Krull domain $T$ is Noetherian.

We consider the following properties of an extension of Krull domains.

Definitions 2.7. Let $S \hookrightarrow T$ be an extension of Krull domains. 
(1) We say that the extension $S \hookrightarrow T$ is weakly flat, or that $T$ is weakly flat over $S$, if every height-one prime ideal $P$ of $S$ with $P T \neq T$ satisfies $P T \cap S=P$.

(2) We say that the extension $S \hookrightarrow T$ is height-one preserving, or that $T$ is a height-one preserving extension of $S$, if for every height-one prime ideal $P$ of $S$ with $P T \neq T$ there exists a height-one prime ideal $Q$ of $T$ with $P T \subseteq Q$.

(3) For $d \in \mathbb{N}$, we say that $\varphi: S \hookrightarrow T$ satisfies $L F_{d}$ (locally flat in height $d$ ), if, for each $P \in \operatorname{Spec} T$ with ht $P \leq d$, the composite map $S \rightarrow T \rightarrow T_{P}$ is flat.

The condition $\mathrm{LF}_{1}$ is equivalent to weak flatness. If $\operatorname{dim} T \leq d$, then the condition $\mathrm{LF}_{d}$ is equivalent to flatness. Proposition 2.8 demonstrates the relevance of the weak flatness property for an extension of Krull domains.

Proposition 2.8. [4, Corollary 12.4] Let $\varphi: S \hookrightarrow T$ be an extension of Krull domains and let $F$ denote the field of fractions of $S$.

(1) Assume that $P T \neq T$ for every height-one prime ideal $P$ of $S$. Then $S \hookrightarrow T$ is weakly flat $\Longleftrightarrow S=F \cap T$.

(2) If $S \hookrightarrow T$ is weakly flat, then $\varphi$ is height-one preserving and, moreover, for every height-one prime ideal $P$ of $S$ with $P T \neq T$, there is a height-one prime ideal $Q$ of $T$ with $Q \cap S=P$.

Theorem 2.9 states that weak flatness of the map $\varphi$ of Equation 1.4. 0 is equivalent to equality of the intersection domain $A$ with its approximation domain $B$.

Theorem 2.9. [4, Theorem 21.14] Assume the notation of Setting[2.2. Thus (T, n) is a local Krull domain with field of fractions $F$, and $y \in \mathbf{n}$ is such that the (y)-adic completion $\left(T^{*}, \mathbf{n}^{*}\right)$ of $T$ is an analytically normal Noetherian local domain and $T=T^{*} \cap F$. For elements $\tau_{1}, \ldots, \tau_{s} \in \mathbf{n}^{*}$ that are algebraically independent over $T$, the following are equivalent:

(1) The intersection domain $A$ is equal to its approximation domain $B$.

(2) The map $\varphi: T\left[\tau_{1}, \ldots, \tau_{s}\right] \longrightarrow T^{*}[1 / y]$ is weakly flat.

(3) The map $B \longrightarrow T^{*}[1 / y]$ is weakly flat.

(4) The map $B \longrightarrow T^{*}$ is weakly flat.

\section{Primarily limit-intersecting Elements}

In this section, we establish the existence of primarily limit-intersecting elements over countable excellent normal local domains.

We use Corollary 3.2 and Lemma 3.3 in the proof of Theorem 3.8 . 
Theorem 3.1. [4, Theorem 11.3] Let $(R, \mathbf{m}),(S, \mathbf{n})$ and $(T, \ell)$ be Noetherian local rings, and assume there exist local homomorphisms:

$$
R \longrightarrow S \longrightarrow T,
$$

such that

(i) $R \rightarrow T$ is flat and $T / \mathbf{m} T$ is Cohen-Macaulay.

(ii) $R \rightarrow S$ is flat and $S / \mathbf{m} S$ is a regular local ring.

Then the following statements are equivalent:

(1) $S \rightarrow T$ is flat.

(2) For each prime ideal $\mathbf{w}$ of $T$, we have $h t(\mathbf{w}) \geq h t(\mathbf{w} \cap S)$.

(3) For each prime ideal $\mathbf{w}$ of $T$ such that $\mathbf{w}$ is minimal over $\mathbf{n} T$, we have $\operatorname{ht}(\mathbf{w}) \geq \operatorname{ht}(\mathbf{n})$.

Since flatness is a local property, the following corollary is immediate.

Corollary 3.2. Let $R, S$ and $T$ be Noetherian rings, and assume there exist ring homomorphisms $R \rightarrow S \rightarrow T$. If the map $R \rightarrow T$ is flat with Cohen-Macaulay fibers and the map $R \rightarrow S$ is flat with regular fibers, then the following two statements are equivalent:

(1) The map $S \rightarrow T$ is flat,

(2) For each prime ideal $P$ of $T$, we have $\operatorname{ht}(P) \geq \operatorname{ht}(P \cap S)$.

To establish the existence of primarily limit-intersecting elements, we use the following prime avoidance lemma; see the articles [3], [11, [13] and the book [5, Lemma 14.2] for other prime avoidance results involving countably infinitely many prime ideals.

Lemma 3.3. [4, Lemma 22.10] Let $(T, \mathbf{n})$ be a Noetherian local domain that is complete in the (y)-adic topology, where $y$ is a nonzero element of $\mathbf{n}$. Let $\mathcal{U}$ be a countable set of prime ideals of $T$ such that $y \notin P$ for each $P \in \mathcal{U}$, and fix an arbitrary element $t \in \mathbf{n} \backslash \mathbf{n}^{2}$. Then there exists an element $a \in y^{2} T$ such that $t-a \notin \bigcup\{P: P \in \mathcal{U}\}$.

Proof. We may assume there are no inclusion relations among the $P \in \mathcal{U}$. We enumerate the prime ideals in $\mathcal{U}$ as $\left\{P_{i}\right\}_{i=1}^{\infty}$. We choose $b_{2} \in T$ so that $t-b_{2} y \notin P_{1}$ as follows: (i) if $t \in P_{1}$, let $b_{2}=1$. Since $y \notin P_{1}$, we have $t-y^{2} \notin P_{1}$. (ii) if $t \notin P_{1}$, let $b_{2}$ be a nonzero element of $P_{1}$. Then $t-b_{2} y^{2} \notin P_{1}$. Assume by induction that we have found $b_{2}, \ldots, b_{n}$ in $T$ such that

$$
t-c y^{2}:=t-b_{2} y^{2}-\cdots-b_{n} y^{n} \notin P_{1} \cup \cdots \cup P_{n-1} .
$$

We choose $b_{n+1} \in T$ so that $t-c y^{2}-b_{n+1} y^{n+1} \notin \bigcup_{i=1}^{n} P_{i}$ as follows: (i) if $t-c y^{2} \in$ $P_{n}$, let $b_{n+1} \in\left(\prod_{i=1}^{n-1} P_{i}\right) \backslash P_{n}$. (ii) if $t-c y^{2} \notin P_{n}$, let $b_{n+1}$ be any nonzero 
element in $\prod_{i=1}^{n} P_{i}$. Hence in either case there exists $b_{n+1} \in T$ so that

$$
t-b_{2} y^{2}-\cdots-b_{n+1} y^{n+1} \notin P_{1} \cup \cdots \cup P_{n} .
$$

Since $T$ is complete in the $(y)$-adic topology, the Cauchy sequence

$$
\left\{b_{2} y^{2}+\cdots+b_{n} y^{n}\right\}_{n=2}^{\infty}
$$

has a limit $a \in \mathbf{n}^{2}$. Since $T$ is Noetherian and local, every ideal of $T$ is closed in the $(y)$-adic topology. Hence, for each integer $n \geq 2$, we have

$$
t-a=\left(t-b_{2} y^{2}-\cdots-b_{n} y^{n}\right)-\left(b_{n+1} y^{n+1}+\cdots\right),
$$

where $t-b_{2} y^{2}-\cdots-b_{n} y^{n} \notin P_{n-1}$ and $\left(b_{n+1} y^{n+1}+\cdots\right) \in P_{n-1}$. We conclude that $t-a \notin \bigcup_{i=1}^{\infty} P_{i}$.

\section{The existence of one primarily limit-intersecting element.}

We use the following setting to describe necessary and sufficient conditions for an element to be primarily limit-intersecting.

Setting 3.4. Let $(R, \mathbf{m})$ be a $d$-dimensional excellent normal local domain with $d \geq 2$, let $y$ be a nonzero element of $\mathbf{m}$ and let $R^{*}$ denote the $(y$ )-adic completion of $R$. Let $t$ be a variable over $R$, let $S:=R[t]_{(\mathbf{m}, t)}$, and let $S^{*}$ denote the $I$-adic completion of $S$, where $I:=(y, t) S$. Then $S^{*}=R^{*}[[t]]$ is a $(d+1)$-dimensional normal Noetherian local domain with maximal ideal $\mathbf{n}^{*}:=(\mathbf{m}, t) S^{*}$. For each element $a \in y^{2} S^{*}$, we have $S^{*}=R^{*}[[t]]=R^{*}[[t-a]]$ since $R^{*}$ is complete in the (a)-adic topology. Let $\lambda_{a}: S^{*} \rightarrow R^{*}$ denote the canonical homomorphism $S^{*} \rightarrow S^{*} /(t-a) S^{*}=R^{*}$, and let $\tau_{a}=\lambda_{a}(t)=\lambda_{a}(a)$. Consider the set

$$
\mathcal{U}:=\left\{P^{*} \in \operatorname{Spec} S^{*} \mid \operatorname{ht}\left(P^{*} \cap S\right)=\operatorname{ht} P^{*}, \text { and } y \notin P^{*}\right\} .
$$

Since $S \hookrightarrow S^{*}$ is flat and thus satisfies the going-down property, the set $\mathcal{U}$ can also be described as the set of all $P^{*} \in \operatorname{Spec} S^{*}$ such that $y \notin P^{*}$ and $P^{*}$ is minimal over $P S^{*}$ for some $P \in \operatorname{Spec} S$; see [6, Theorem 15.1]

Theorem 3.5. With the notation of Setting 3.4, the element $\tau_{a}$ is primarily limitintersecting in $y$ over $R$ if and only if $t-a \notin \bigcup\left\{P^{*} \mid P^{*} \in \mathcal{U}\right\}$.

Proof. Consider the commutative diagram:

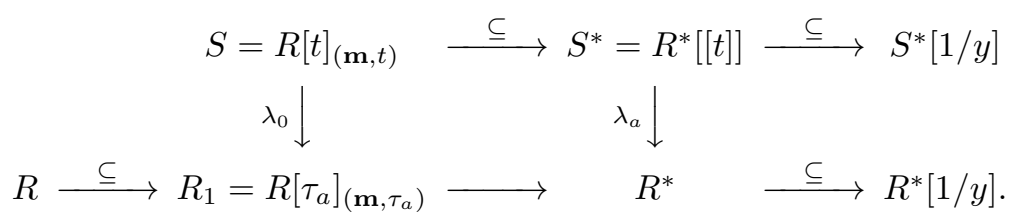

Diagram 3.5.0

The map $\lambda_{0}$ denotes the restriction of $\lambda_{a}$ to $S$. 
Assume that $\tau_{a}$ is primarily limit-intersecting in $y$ over $R$. Then $\tau_{a}$ is algebraically independent over $R$ and $\lambda_{0}$ is an isomorphism. If $t-a \in P^{*}$ for some $P^{*} \in \mathcal{U}$, we prove that $\varphi: R_{1} \rightarrow R^{*}[1 / y]$ is not flat. Let $Q^{*}:=\lambda_{a}\left(P^{*}\right)$. We have ht $Q^{*}=$ ht $P^{*}-1$, and $y \notin P^{*}$ implies $y \notin Q^{*}$. Let $P:=P^{*} \cap S$ and $Q:=Q^{*} \cap R_{1}$. Commutativity of Diagram 3.5.0 and $\lambda_{0}$ an isomorphism imply that ht $P=$ ht $Q$. Since $P^{*} \in \mathcal{U}$, we have ht $P=\operatorname{ht} P^{*}$. It follows that ht $Q>$ ht $Q^{*}$. This implies that $\varphi: R_{1} \rightarrow R^{*}[1 / y]$ is not flat.

For the converse, assume that $t-a \notin \bigcup\left\{P^{*} \mid P^{*} \in \mathcal{U}\right\}$. Since $a \in y^{2} S^{*}$ and $S^{*}$ is complete in the $(y, t)$-adic topology, we have $S^{*}=R^{*}[[t]]=R^{*}[[t-a]]$. Thus

$$
\mathfrak{p}:=\operatorname{ker}\left(\lambda_{a}\right)=\left(t-\tau_{a}\right) S^{*}=(t-a) S^{*}
$$

is a height-one prime ideal of $S^{*}$. Since $y \in R$ and $\mathfrak{p} \cap R=(0)$, we have $y \notin \mathfrak{p}$.

Since $t-a$ is outside every element of $\mathcal{U}$, we have $\mathfrak{p} \notin \mathcal{U}$. Since $\mathfrak{p}$ does not fit the condition of $\mathcal{U}$, we have $\operatorname{ht}(\mathfrak{p} \cap S) \neq \mathrm{ht} \mathfrak{p}=1$, and so, by the faithful flatness of $S \hookrightarrow S^{*}, \mathfrak{p} \cap S=(0)$. Therefore the map $\lambda_{0}: S \rightarrow R_{1}$ has trivial kernel, and so $\lambda_{0}$ is an isomorphism. Thus $\tau_{a}$ is algebraically independent over $R$.

Since $R$ is excellent and $R_{1}$ is a localized polynomial $\operatorname{ring}$ over $R$, the hypotheses of Corollary 3.2 are satisfied for the extension $R \hookrightarrow R_{1} \hookrightarrow R^{*}[1 / y]$. It follows that the element $\tau_{a}$ is primarily limit-intersecting in $y$ over $R$ if $\operatorname{ht}\left(Q_{1}^{*} \cap R_{1}\right) \leq \operatorname{ht} Q_{1}^{*}$ for every prime ideal $Q_{1}^{*} \in \operatorname{Spec}\left(R^{*}[1 / y]\right)$, or, equivalently, if, for every $Q^{*} \in \operatorname{Spec} R^{*}$ with $y \notin Q^{*}$, we have ht $\left(Q^{*} \cap R_{1}\right) \leq \mathrm{ht} Q^{*}$. Thus, to complete the proof of Theorem 3.5, it suffices to prove Claim 3.6 .

Claim 3.6. For every prime ideal $Q^{*} \in \operatorname{Spec} R^{*}$ with $y \notin Q^{*}$, we have

$$
\operatorname{ht}\left(Q^{*} \cap R_{1}\right) \leq \operatorname{ht} Q^{*} .
$$

Proof of Claim 3.6. Since $\operatorname{dim} R^{*}=d$ and $y \notin Q^{*}$, we have ht $Q^{*}=r \leq d-1$. Since the map $R \hookrightarrow R^{*}$ is flat, we have ht $\left(Q^{*} \cap R\right) \leq$ ht $Q^{*}=r$. Suppose that $Q:=Q^{*} \cap R_{1}$ has height at least $r+1 \mathrm{in} \operatorname{Spec} R_{1}$. Since $R_{1}$ is a localized polynomial ring in one variable over $R$ and $\operatorname{ht}(Q \cap R) \leq r$, we have ht $(Q)=r+1$. Let $P:=\lambda_{0}^{-1}(Q) \in \operatorname{Spec} S$. Then ht $P=r+1$ and $y \notin P$.

Let $P^{*}:=\lambda_{a}^{-1}\left(Q^{*}\right)$. Since the prime ideals of $S^{*}$ that contain $t-a$ and have height $r+1$ are in one-to-one correspondence with the prime ideals of $R^{*}$ of height $r$, we have ht $P^{*}=r+1$. By the commutativity of the diagram, we also have $y \notin P^{*}$ and $P \subseteq P^{*} \cap S$, and so

$$
r+1=\operatorname{ht} P \leq \operatorname{ht}\left(P^{*} \cap S\right) \leq \operatorname{ht} P^{*}=r+1,
$$

where the last inequality holds because the map $S \hookrightarrow S^{*}$ is flat. It follows that $P=P^{*} \cap S$, and so $P^{*} \in \mathcal{U}$. This contradicts the fact that $t-a \notin P_{1}^{*}$ for each $P_{1}^{*} \in \mathcal{U}$. Thus we have $\operatorname{ht}\left(Q^{*} \cap R_{1}\right) \leq r=\mathrm{ht} Q^{*}$, as asserted in Claim 3.6. 
This completes the proof of Theorem 3.5 .

Theorem 3.5 yields a necessary and sufficient condition for an element of $R^{*}$ that is algebraically independent over $R$ to be primarily limit-intersecting in $y$ over $R$.

Remarks 3.7. Assume notation as in Setting 3.4.

(1) For each $a \in y^{2} S^{*}$ as in Setting 3.4, we have $(t-a) S^{*}=\left(t-\tau_{a}\right) S^{*}$. Hence $t-a \notin \bigcup\left\{P^{*} \mid P^{*} \in \mathcal{U}\right\} \Longleftrightarrow t-\tau_{a} \notin \bigcup\left\{P^{*} \mid P^{*} \in \mathcal{U}\right\}$.

(2) If $a \in R^{*}$, then the commutativity of Diagram 3.5.0 implies that $\tau_{a}=a$.

(3) For $\tau \in R^{*}$, we have $\tau=b_{0}+b_{1} y+\tau^{\prime}$, where $b_{0}$ and $b_{1}$ are in $R$ and $\tau^{\prime} \in y^{2} R^{*}$.

(a) The rings $R[\tau]$ and $R\left[\tau^{\prime}\right]$ are equal. Hence $\tau$ is primarily limit-intersecting in $y$ over $R$ if and only if $\tau^{\prime}$ is primarily limit-intersecting in $y$ over $R$.

(b) Assume $\tau \in R^{*}$ is algebraically independent over $R$. Then $\tau$ is primarily limit-intersecting in $y$ over $R$ if and only if $t-\tau^{\prime} \notin \bigcup\left\{P^{*} \mid P^{*} \in \mathcal{U}\right\}$. Item $3 \mathrm{~b}$ follows from Theorem 3.5 by setting $a=\tau^{\prime}$ and applying item $3 \mathrm{a}$ and item 2.

We use Theorem 3.5 and Lemma 3.3 to prove Theorem 3.8 .

Theorem 3.8. Let $(R, \mathbf{m})$ be a countable excellent normal local domain of dimension $d \geq 2$, let $y$ be a nonzero element in $\mathbf{m}$, and let $R^{*}$ denote the $(y)$-adic completion of $R$. Then there exists an element $\tau \in y R^{*}$ that is primarily limit-intersecting in $y$ over $R$.

Proof. As in Setting 3.4, let

$$
\mathcal{U}:=\left\{P^{*} \in \operatorname{Spec} S^{*} \mid \operatorname{ht}\left(P^{*} \cap S\right)=\operatorname{ht} P^{*}, \text { and } y \notin P^{*}\right\} .
$$

Since the ring $S$ is countable and Noetherian, the set $\mathcal{U}$ is countable. Lemma 3.3 implies that there exists an element $a \in y^{2} S^{*}$ such that $t-a \notin \bigcup\left\{P^{*} \mid P^{*} \in \mathcal{U}\right\}$. By Theorem 3.5, the element $\tau_{a}$ is primarily limit-intersecting in $y$ over $R$.

\section{The existence of more primarily limit-intersecting elements.}

To establish the existence of more than one primarily limit-intersecting element we use the following setting.

Setting 3.9. Let $(R, \mathbf{m})$ be a $d$-dimensional excellent normal local domain, let $y$ be a nonzero element of $\mathbf{m}$ and let $R^{*}$ denote the $(y)$-adic completion of $R$. Let $t_{1}, \ldots, t_{n+1}$ be indeterminates over $R$, and let $S_{n}$ and $S_{n+1}$ denote the localized polynomial rings

$$
S_{n}:=R\left[t_{1}, \ldots, t_{n}\right]_{\left(\mathbf{m}, t_{1}, \ldots, t_{n}\right)} \quad \text { and } \quad S_{n+1}:=R\left[t_{1}, \ldots, t_{n+1}\right]_{\left(\mathbf{m}, t_{1}, \ldots, t_{n+1}\right)} .
$$


Let $S_{n}^{*}$ denote the $I_{n}$-adic completion of $S_{n}$, where $I_{n}:=\left(y, t_{1}, \ldots, t_{n}\right) S_{n}$. Then $S_{n}^{*}=R^{*}\left[\left[t_{1}, \ldots, t_{n}\right]\right]$ is a $(d+n)$-dimensional normal Noetherian local domain with maximal ideal $\mathbf{n}^{*}=\left(\mathbf{m}, t_{1}, \ldots, t_{n}\right) S_{n}^{*}$. Assume that $\tau_{1}, \ldots, \tau_{n} \in y R^{*}$ are primarily limit-intersecting in $y$ over $R$, and define $\lambda: S_{n}^{*} \rightarrow R^{*}$ to be the $R^{*}$-algebra homomorphism such that $\lambda\left(t_{i}\right)=\tau_{i}$, for $1 \leq i \leq n$.

Since $S_{n}^{*}=R^{*}\left[\left[t_{1}-\tau_{1}, \ldots, t_{n}-\tau_{n}\right]\right]$, we have $\mathbf{p}_{n}:=\operatorname{ker} \lambda=\left(t_{1}-\tau_{1}, \ldots, t_{n}-\tau_{n}\right) S_{n}^{*}$. Consider the commutative diagram:

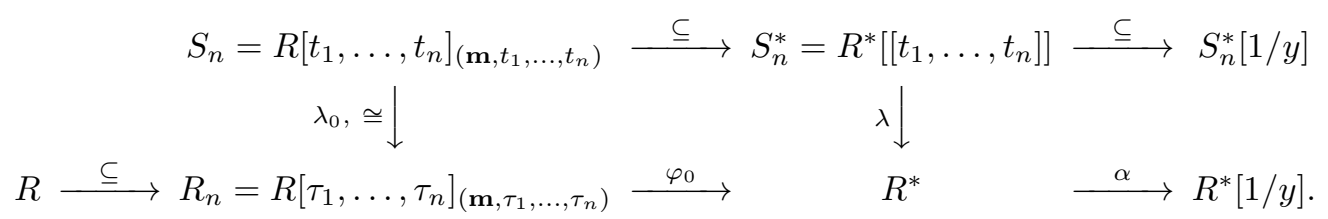

Let $S_{n+1}^{*}$ denote the $I_{n+1}$-adic completion of $S_{n+1}$, where $I_{n+1}:=\left(y, t_{1}, \ldots, t_{n+1}\right) S_{n+1}$. For each element $a \in y^{2} S_{n+1}^{*}$, we have

$$
S_{n+1}^{*}=S_{n}^{*}\left[\left[t_{n+1}\right]\right]=S_{n}^{*}\left[\left[t_{n+1}-a\right]\right] .
$$

Let $\lambda_{a}: S_{n+1}^{*} \rightarrow R^{*}$ denote the composition

$$
S_{n+1}^{*}=S_{n}^{*}\left[\left[t_{n+1}\right]\right] \longrightarrow \frac{S_{n}^{*}\left[\left[t_{n+1}\right]\right]}{\left(t_{n+1}-a\right)}=S_{n}^{*} \stackrel{\lambda}{\longrightarrow} R^{*},
$$

and let $\tau_{a}:=\lambda_{a}\left(t_{n+1}\right)=\lambda_{a}(a)$. We have $\operatorname{ker} \lambda_{a}=\left(\mathbf{p}_{n}, t_{n+1}-a\right) S_{n+1}^{*}$. Consider the commutative diagram

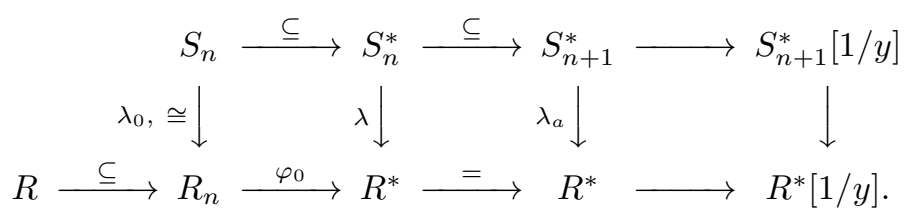

Diagram $3.9,2$

Let

$\mathcal{U}:=\left\{P^{*} \in \operatorname{Spec} S_{n+1}^{*} \mid P^{*} \cap S_{n+1}=P, y \notin P\right.$ and $P^{*}$ is minimal over $\left.\left(P, \mathbf{p}_{n}\right) S_{n+1}^{*}\right\}$.

Notice that $y \notin P^{*}$ for each $P^{*} \in \mathcal{U}$, since $y \in R$ implies $\lambda_{a}(y)=y$.

Theorem 3.10. With the notation of Setting 3.9, the elements $\tau_{1}, \ldots, \tau_{n}, \tau_{a}$ are primarily limit-intersecting in $y$ over $R$ if and only if $t_{n+1}-a \notin \bigcup\left\{P^{*} \mid P^{*} \in \mathcal{U}\right\}$.

Proof. Assume that $\tau_{1}, \ldots, \tau_{n}, \tau_{a}$ are primarily limit-intersecting in $y$ over $R$. Then $\tau_{1}, \ldots, \tau_{n}, \tau_{a}$ are algebraically independent over $R$. Consider the following commutative diagram:

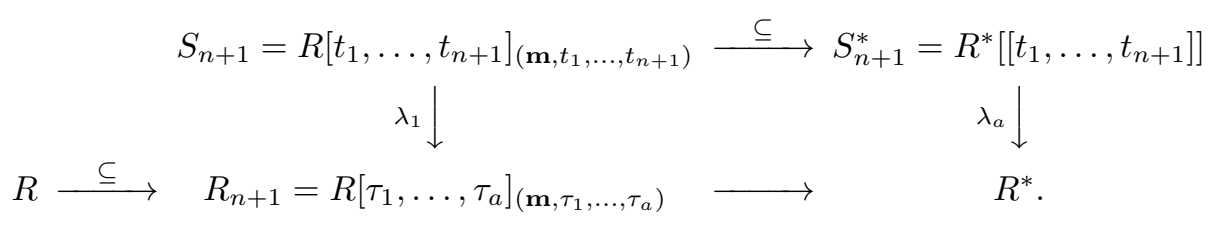


Diagram 3.10

The map $\lambda_{1}$ is the restriction of $\lambda_{a}$ to $S_{n+1}$, and is an isomorphism since $\tau_{1}, \ldots, \tau_{n}, \tau_{a}$ are algebraically independent over $R$.

If $t_{n+1}-a \in P^{*}$ for some $P^{*} \in \mathcal{U}$, we prove that $\varphi: R_{n+1} \rightarrow R^{*}[1 / y]$ is not flat, a contradiction to our assumption that $\tau_{1}, \ldots, \tau_{n}, \tau_{a}$ are primarily limit-intersecting. Since $P^{*} \in \mathcal{U}$, we have $\mathbf{p}_{n} \subset P^{*}$. Then $t_{n+1}-a \in P^{*}$ implies $\operatorname{ker} \lambda_{a} \subset P^{*}$. Let $\lambda_{a}\left(P^{*}\right):=Q^{*}$. Then $\lambda_{a}^{-1}\left(Q^{*}\right)=P^{*}$ and ht $P^{*}=n+1+$ ht $Q^{*}$. Since $P^{*} \in \mathcal{U}$, we have $y \notin P^{*}$. The commutativity of Diagram 3.10 0 implies that $y \notin Q^{*}$. Let $P:=P^{*} \cap S_{n+1}$ and let $Q:=Q^{*} \cap R_{n+1}$. Commutativity of Diagram [3.10]0 and $\lambda_{0}$ an isomorphism imply that ht $P=$ ht $Q$. Since $P^{*}$ is a minimal prime of $\left(P, \mathbf{p}_{n}\right) S_{n+1}^{*}, \mathbf{p}_{n}$ is $n$-generated, and $S_{n+1}^{*}$ is Noetherian and catenary, we have ht $P^{*} \leq$ ht $P+n$. Hence ht $P \geq$ ht $P^{*}-n$. Thus

$$
\text { ht } Q=\text { ht } P \geq \text { ht } P^{*}-n=\text { ht } Q^{*}+n+1-n=\text { ht } Q^{*}+1 .
$$

The fact that ht $Q>$ ht $Q^{*}$ implies that the map $R_{n+1} \rightarrow R^{*}[1 / y]$ is not flat. This proves the forward direction.

For the converse, we have

$$
\text { Assumption 3.10,1: } \quad t_{n+1}-a \notin \bigcup\left\{P^{*} \mid P^{*} \in \mathcal{U}\right\} \text {. }
$$

Since $\lambda_{a}: S_{n+1}^{*} \rightarrow R^{*}$ is an extension of $\lambda: S_{n}^{*} \rightarrow R^{*}$ as in Diagram 3.9. 2, we have $\operatorname{ker} \lambda_{a} \cap S_{n}=(0)$. Let $\mathbf{p}:=\left(t_{n+1}-a\right) S_{n+1}^{*}=\left(t_{n+1}-\tau_{a}\right) S_{n+1}^{*}$. As in Equation 3.9.1, we have

$$
S_{n+1}^{*}=R^{*}\left[\left[t_{1}, \ldots, t_{n+1}\right]\right]=R^{*}\left[\left[t_{1}-\tau_{1}, \ldots, t_{n}-\tau_{n}, t_{n+1}-a\right]\right] .
$$

Thus $P^{*}:=\left(\mathbf{p}_{n}, \mathbf{p}\right) S_{n+1}^{*}$ is a prime ideal of height $n+1$ and $P^{*} \cap R^{*}=(0)$. It follows that $y \notin P^{*}$. We show that $P^{*} \cap S_{n+1}=(0)$. Assume that $P=P^{*} \cap S_{n+1} \neq(0)$. Since ht $P *=n+1, P^{*}$ is minimal over $\left(P, \mathbf{p}_{n}\right) S_{n+1}^{*}$, and so $P^{*} \in \mathcal{U}$, a contradiction to Assumption 3.10, 1. Therefore $P^{*} \cap S_{n+1}=(0)$. It follows that $\mathbf{p} \cap S_{n+1}=(0)$ since $\mathbf{p} \subset P^{*}$. Thus ker $\lambda_{1}=(0)$, and so $\lambda_{1}$ in Diagram 3.10,0 is an isomorphism. Therefore $\tau_{a}$ is algebraically independent over $R_{n}$.

Since $R$ is excellent and $R_{n+1}$ is a localized polynomial ring in $n+1$ variables over $R$, the hypotheses of Corollary 3.2 are satisfied for the composition

$$
R \hookrightarrow R_{n+1} \hookrightarrow R^{*}[1 / y] .
$$

It follows that the elements $\tau_{1}, \ldots, \tau_{n}, \tau_{a}$ are primarily limit-intersecting in $y$ over $R$ if, for every $Q^{*} \in \operatorname{Spec} R^{*}$ with $y \notin Q^{*}$, we have ht $\left(Q^{*} \cap R_{n+1}\right) \leq \operatorname{ht} Q^{*}$. Thus, to complete the proof of Theorem 3.10 with $\tau_{n+1}=\tau_{a}$, it suffices to prove Claim 3.11

Claim 3.11. Let $Q^{*} \in \operatorname{Spec} R^{*}$ with $y \notin Q^{*}$ and ht $Q^{*}=r$. Then

$$
\operatorname{ht}\left(Q^{*} \cap R_{n+1}\right) \leq r .
$$


Proof of Claim 3.11. Let $Q_{1}:=Q^{*} \cap R_{n+1}$ and let $Q_{0}:=Q^{*} \cap R_{n}$. Suppose ht $Q_{1}>r$. Notice that $r<d$, since $d=\operatorname{dim} R^{*}$ and $y \notin Q^{*}$.

Since $\tau_{1}, \ldots, \tau_{n}$ are primarily limit-intersecting in $y$ over $R$, the extension

$$
R_{n}:=R\left[\tau_{1}, \ldots, \tau_{n}\right]_{\left(\mathbf{m}, \tau_{1}, \ldots, \tau_{n}\right)} \hookrightarrow R^{*}[1 / y]
$$

from Diagram [3.9.2 is flat. Thus ht $Q_{0} \leq r$ and ht $Q_{0} \leq$ ht $L^{*}$ for every prime ideal $L^{*}$ of $R^{*}$ with $Q_{0} R^{*} \subseteq L^{*} \subseteq Q^{*}$. Since $R_{n+1}$ is a localized polynomial ring in the indeterminate $\tau_{a}$ over $R_{n}$, we have that ht $Q_{1} \leq$ ht $Q_{0}+1=r+1$. Thus ht $Q_{1}=r+1$ and ht $Q_{0}=r$. It follows that $Q^{*}$ is a minimal prime of $Q_{0} R^{*}$.

Let $h\left(\tau_{a}\right) \in R_{n}\left[\tau_{a}\right]=R_{n+1}$ be a polynomial in the variable $\tau_{a}$ over the ring $R_{n}$ such that

$$
h\left(\tau_{a}\right) \in\left(Q^{*} \cap R_{n}\left[\tau_{a}\right]\right) \backslash\left(Q^{*} \cap R_{n}\right) R_{n+1} .
$$

It follows that $Q_{1}$ is a minimal prime of the ideal $\left(Q_{0}, h\left(\tau_{a}\right)\right) R_{n+1}$.

With notation from Diagram $3.9,2$, define

$$
P_{0}:=\lambda_{0}^{-1}\left(Q_{0}\right) \text { and } P_{0}^{*}:=\lambda^{-1}\left(Q^{*}\right) .
$$

Since $\lambda_{0}$ is an isomorphism, $P_{0}$ is a prime ideal of $S_{n}$ with ht $P_{0}=r$. Moreover, we have the following:

(1) $P_{0}^{*} \cap S_{n}=P_{0}$ (by commutativity in Diagram 3.92 ),

(2) $y \notin P_{0}^{*}$ (by item 1 ),

(3) $P_{0}^{*}$ is a minimal prime of $\left(P_{0}, \mathbf{p}_{n}\right) S_{n}^{*}$ (since $S_{n}^{*} / \mathbf{p}_{n}=R^{*}$ in Diagram 3.9 , 2 , and $Q^{*}$ is a minimal prime of $Q_{0} R^{*}$,

(4) ht $P_{0}^{*}=n+r$ (by the correspondence between prime ideals of $S_{n}^{*}$ containing $\mathbf{p}_{n}$ and prime ideals of $R^{*}$ ).

Consider the commutative diagram below with the left and right ends identified:

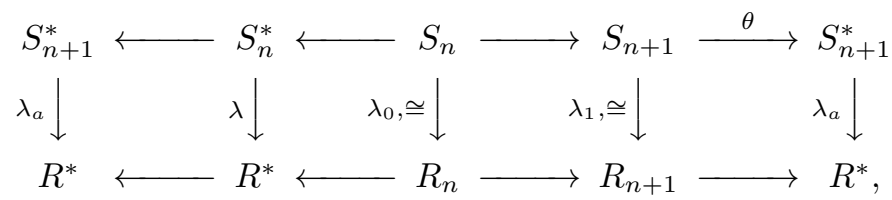

Diagram $3.11,0$

where $\lambda, \lambda_{0}$ and $\lambda_{1}$ are as in Diagrams $3.9,2$ and 3.10 0 , and so $\lambda_{a}$ restricted to $S_{n}^{*}$ is $\lambda$. Let $h\left(t_{n+1}\right)=\lambda_{1}^{-1}\left(h\left(\tau_{a}\right)\right)$ and set

$$
P_{1}:=\lambda_{1}^{-1}\left(Q_{1}\right) \in \operatorname{Spec}\left(S_{n+1}\right), \text { and } P^{*}:=\lambda_{a}^{-1}\left(Q^{*}\right) \in \operatorname{Spec}\left(S_{n+1}^{*}\right) .
$$

Then $P_{1}$ is a minimal prime of $\left(P_{0}, h\left(t_{n+1}\right)\right) S_{n+1}$, since $Q_{1}$ is a minimal prime of $\left(Q_{0}, h\left(\tau_{a}\right)\right) R_{n+1}$. Since $Q_{1} \subseteq Q^{*}$, we have $h\left(t_{n+1}\right) \in P^{*}$ and $P_{1} S_{n+1}^{*} \subseteq P^{*}$ because $\lambda_{a}\left(h\left(t_{n+1}\right)\right)=\lambda_{1}\left(h\left(t_{n+1}\right)\right)=h\left(\tau_{a}\right) \in Q_{1}$ and $\lambda_{a}\left(P_{1}\right)=\lambda_{1}\left(P_{1}\right)=Q_{1}$. By the correspondence between prime ideals of $S_{n+1}^{*}$ containing $\operatorname{ker}\left(\lambda_{a}\right)=\mathbf{p}_{n+1}$ and 
prime ideals of $R^{*}$, we see

$$
\text { ht } P^{*}=\text { ht } Q^{*}+n+1=r+n+1 .
$$

Since $\lambda_{a}\left(P_{0}^{*}\right) \subseteq Q^{*}$, we have $P_{0}^{*} \subseteq P^{*}$, but $h\left(t_{n+1}\right) \notin P_{0}$ implies $h\left(t_{n+1}\right) \notin P_{0}^{*} S_{n+1}^{*}$. Therefore

$$
\left(P_{0}, \mathbf{p}_{n}\right) S_{n+1}^{*} \subseteq P_{0}^{*} S_{n+1}^{*} \subsetneq\left(P_{0}^{*}, h\left(t_{n+1}\right)\right) S_{n+1}^{*} \subseteq P^{*} .
$$

By items 3 and 4 above, ht $P_{0}^{*}=n+r$ and $P_{0}^{*}$ is a minimal prime of $\left(P_{0}, \mathbf{p}_{n}\right) S_{n}^{*}$. Since ht $P^{*}=n+r+1$, it follows that $P^{*}$ is a minimal prime of $\left(P_{0}, h\left(t_{n+1}\right), \mathbf{p}_{n}\right) S_{n+1}^{*}$. Since $\left(P_{0}, h\left(t_{n+1}\right), \mathbf{p}_{n}\right) S_{n+1}^{*} \subseteq\left(P_{1}, \mathbf{p}_{n}\right) S_{n+1}^{*} \subseteq P^{*}$, we have $P^{*}$ is a minimal prime of $\left(P_{1}, \mathbf{p}_{n}\right) S_{n+1}^{*}$. But then, by Assumption 3.10, 1 on $\mathcal{U}$, we have $t_{n+1}-a \notin P^{*}$, a contradiction. This contradiction implies that ht $Q_{1}=r$.

This completes the proof of Claim 3.11 and thus the proof of Theorem 3.10 .

We use Theorem 3.8, Theorem 3.10 and Lemma 3.3 to prove in Theorem 3.12 the existence over a countable excellent normal local domain of dimension at least two of an infinite sequence of primarily limit-intersecting elements.

Theorem 3.12. Let $R$ be a countable excellent normal local domain of dimension $d \geq 2$, let $y$ be a nonzero element in the maximal ideal $\mathbf{m}$ of $R$, and let $R^{*}$ be the (y)-adic completion of $R$. Let $n$ be a positive integer. Then

(1) If the elements $\tau_{1}, \ldots, \tau_{n} \in y R^{*}$ are primarily limit-intersecting in $y$ over $R$, then there exists an element $\tau_{a} \in y R^{*}$ such that $\tau_{1}, \ldots, \tau_{n}, \tau_{a}$ are primarily limit-intersecting in y over $R$.

(2) There exists an infinite sequence $\tau_{1}, \ldots, \tau_{n}, \ldots \in y R^{*}$ of elements that are primarily limit-intersecting in $y$ over $R$.

Proof. By Definition [1.5, item 1 implies item 2; thus it suffices to prove item 1. Theorem 3.8 implies the existence of an element $\tau_{1} \in y R^{*}$ that is primarily limitintersecting in $y$ over $R$. As in Setting 3.9, let

$\mathcal{U}:=\left\{P^{*} \in \operatorname{Spec} S_{n+1}^{*} \mid P^{*} \cap S_{n+1}=P, y \notin P\right.$ and $P^{*}$ is minimal over $\left.\left(P, \mathbf{p}_{n}\right) S_{n+1}^{*}\right\}$.

Since the ring $S_{n+1}$ is countable and Noetherian, the set $\mathcal{U}$ is countable. Lemma 3.3 implies that there exists an element $a \in y^{2} S_{n+1}^{*}$ such that

$$
t_{n+1}-a \notin \bigcup\left\{P^{*} \mid P^{*} \in \mathcal{U}\right\} .
$$

By Theorem 3.10 the elements $\tau_{1}, \ldots, \tau_{n}, \tau_{a}$ are primarily limit-intersecting in $y$ over $R$.

\section{Normal Noetherian domains that are not excellent.}


Using Theorem 3.8, we establish in Theorem 3.13, for every countable excellent normal local domain $R$ of dimension $d \geq 2$, the existence of a primarily limitintersecting element $\eta \in y R^{*}$ such that the constructed Noetherian domain

$$
B=A=R^{*} \cap \mathcal{Q}(R[\eta])
$$

is not a Nagata domain and hence is not excellent 3

Theorem 3.13. Let $R$ be a countable excellent normal local domain of dimension $d \geq 2$, let $y$ be a nonzero element in the maximal ideal $\mathbf{m}$ of $R$, and let $R^{*}$ be the (y)-adic completion of $R$. There exists an element $\eta \in y R^{*}$ such that

(1) $\eta$ is primarily limit-intersecting in $y$ over $R$.

(2) The associated intersection domain $A:=R^{*} \cap \mathcal{Q}(R[\eta])$ is equal to its approximation domain $B$.

(3) The ring $A$ has a height-one prime ideal $\mathbf{p}$ such that $R^{*} / \mathbf{p} R^{*}$ is not reduced. Thus the integral domain $A=B$ associated to $\eta$ is a normal Noetherian local domain that is not a Nagata domain and hence is not excellent.

Proof. Since $\operatorname{dim} R \geq 2$, there exists $x \in \mathbf{m}$ such that $\mathrm{ht}(x, y) R=2$. By Theorem 3.8 there exists $\tau \in y R^{*}$ such that $\tau$ is primarily limit-intersecting in $y$ over $R$. Hence the extension $R[\tau] \longrightarrow R^{*}[1 / y]$ is flat. Let $n \in \mathbb{N}$ with $n \geq 2$, and let $\eta:=(x+\tau)^{n}$. Since $\tau$ is algebraically independent over $R$, the element $\eta$ is also algebraically independent over $R$. Moreover, the polynomial ring $R[\tau]$ is a free $R[\eta]$ module with $1, \tau, \ldots, \tau^{n-1}$ as a free module basis. Hence the map $R[\eta] \longrightarrow R^{*}[1 / y]$ is flat. It follows that $\eta$ is primarily limit-intersecting in $y$ over $R$. Therefore the intersection domain $A:=R^{*} \cap \mathcal{Q}(R[\eta])$ is equal to its associated approximation domain $B$ and is a normal Noetherian domain with $(y)$-adic completion $R^{*}$. Since $\eta$ is a prime element of the polynomial ring $R[\eta]$ and $B[1 / y]$ is a localization of $R[\eta]$, it follows that $\mathbf{p}:=\eta B$ is a height-one prime ideal of $B$. Since $\tau \in R^{*}$, and $\eta=(x+\tau)^{n}$, the ring $R^{*} / \mathbf{p} R^{*}$ contains nonzero nilpotent elements. Since $\widehat{R}=\widehat{B}$ is faithfully flat over $R^{*}$, it follows that $\widehat{B} / \mathbf{p} \widehat{B}$ has nonzero nilpotent elements. Since a Nagata local domain is analytically unramified, it follows that the normal Noetherian domain $B$ is not a Nagata ring, [6, page 264] or [8, (32.2)].

\section{Other Results AND EXAmples using the CONSTRUCTION}

We use the following notation for the beginning of this section, and make several remarks concerning properties of and relationships among the integral domains being considered.

\footnotetext{
3 "Nagata" is defined after Question [1.2 see also [4, Definitions 2.3.1, 3.28], [6] pages 264, 260].
} 
Notation and Remarks 4.1. Let $k$ be a field, let $x$ and $y$ be indeterminates over $k$, and let

$$
\sigma:=\sum_{i=1}^{\infty} a_{i} x^{i} \in x k[[x]] \quad \text { and } \quad \tau:=\sum_{i=1}^{\infty} b_{i} y^{i} \in y k[[y]]
$$

be formal power series that are algebraically independent over the fields $k(x)$ and $k(y)$, respectively. Let $R:=k[x, y]_{(x, y)}$, and let $\sigma_{n}, \tau_{n}$ be the $n^{\text {th }}$ endpieces of $\sigma, \tau$ respectively. Define

$$
\begin{aligned}
C_{n} & :=k\left[x, \sigma_{n}\right]_{\left(x, \sigma_{n}\right)}, \quad C:=k(x, \sigma) \cap k[[x]]=\underline{\lim }\left(C_{n}\right)=\bigcup_{n=1}^{\infty} C_{n} ; \\
D_{n} & :=k\left[y, \tau_{n}\right]_{\left(y, \tau_{n}\right)}, \quad D:=k(y, \tau) \cap k[[y]]=\underline{\lim }\left(D_{n}\right)=\bigcup_{n=1}^{\infty} D_{n} ; \\
U_{n} & :=k\left[x, y, \sigma_{n}, \tau_{n}\right], \quad U:=\underline{\lim _{\longrightarrow}} U_{n}=\bigcup_{n=1}^{\infty} U_{n} ; \\
B_{n} & :=k\left[x, y, \sigma_{n}, \tau_{n}\right]_{\left(x, y, \sigma_{n}, \tau_{n}\right)} \quad B:=\underline{\lim _{\longrightarrow}}\left(B_{n}\right)=\bigcup_{n=1}^{\infty} B_{n} ; \\
A & :=k(x, y, \sigma, \tau) \cap k[[x, y]] .
\end{aligned}
$$

Since $k[[x, y]]$ is the $(x, y)$-adic completion of the Noetherian ring $R$, the ring $k[[x, y]]=\widehat{R}$ is faithfully flat over $R$. Hence we have

$$
(x, y)^{n} k[[x, y]] \cap R=(x, y)^{n} R
$$

for each $n \in \mathbb{N}$.

The relationships

$$
\sigma_{n}=-x a_{n+1}+x \sigma_{n+1} \quad \text { and } \quad \tau_{n}=-y b_{n+1}+y \tau_{n+1}
$$

among the endpieces imply for each positive integer $n$ the inclusions

$$
C_{n} \subset C_{n+1}, \quad D_{n} \subset D_{n+1}, \quad \text { and } \quad B_{n} \subset B_{n+1} .
$$

Moreover, for each of these inclusions we have birational domination of the larger local ring over the smaller, and the local rings $C_{n}, D_{n}, B_{n}$ are all dominated by $k[[x, y]]=\widehat{R}$.

Since $\left(x, y, \sigma_{n}, \tau_{n}\right) U_{n}$ is a maximal ideal of $U_{n}$ that is contained in $(x, y) U$, a proper ideal of $U$, it follows that $(x, y) U \cap U_{n}=\left(x, y, \sigma_{n}, \tau_{n}\right) U_{n}$. Since $B_{n}$ is the localization of the polynomial ring $U_{n}$ at the maximal ideal $\left(x, y, \sigma_{n}, \tau_{n}\right) U_{n}$, we have $(x, y) B \cap B_{n}=\left(x, y, \sigma_{n}, \tau_{n}\right) B$ for each $n \in \mathbb{N}$.

We have $\sigma_{n+1} \in U_{n}\left[\frac{1}{x}\right] \subseteq U_{n}\left[\frac{1}{x y}\right]$ and $\tau_{n+1} \in U_{n}\left[\frac{1}{y}\right] \subseteq U_{n}\left[\frac{1}{x y}\right]$, for each $n \in \mathbb{N}$. Hence $U_{n+1} \subseteq U_{n}\left[\frac{1}{x y}\right]$, and $U \subseteq U_{n}\left[\frac{1}{x y}\right]$, and $U_{n}\left[\frac{1}{x y}\right]=U\left[\frac{1}{x y}\right]$. 
The rings $C$ and $D$ are rank-one discrete valuation domains that are directed unions of two-dimensional regular local domains. Each of the rings $B_{n}$ is a fourdimensional regular local domain that is a localized polynomial ring over the field $k$. Thus $B$ is the directed union of a chain of four-dimensional regular local domains.

Theorem 4.2. Assume the setting of Notation 4.1. Then the ring $A$ is a twodimensional regular local domain that birationally dominates the ring $B ; A$ has maximal ideal $(x, y) A$ and completion $\widehat{A}=k[[x, y]]$. Moreover we have:

(1) The rings $U$ and $B$ are UFDs,

(2) $B$ is a local Krull domain with maximal ideal $\mathbf{n}=(x, y) B$,

(3) The dimension of $B$ is either 2 or 3, depending on the choice of $\sigma$ and $\tau$,

(4) $B$ is Hausdorff in the topology defined by the powers of $\mathbf{n}$,

(5) The $\mathbf{n}$-adic completion $\widehat{B}$ of $B$ is canonically isomorphic to $k[[x, y]]$, and

(6) The following statements are equivalent:

(a) $B=A$.

(b) $B$ is a two-dimensional regular local domain.

(c) B is Noetherian.

(d) Every finitely generated ideal of $B$ is closed in the $\mathbf{n}$-adic topology on $B$.

(e) Every principal ideal of $B$ is closed in the $\mathbf{n}$-adic topology on $B$.

Proof. The assertions about $A$ follow from a theorem of Valabrega [12]; see [4, Proposition 4.13]. Since $U_{0}$ has field of fractions $k(x, y, \sigma, \tau)=\mathcal{Q}(A)$ and $U_{0} \subseteq$ $B \subseteq A$, the extension $B \hookrightarrow A$ is birational. Since $B$ is the directed union of the four-dimensional regular local domains $B_{n}$ and $(x, y) B \cap B_{n}=\left(x, y, \sigma_{n}, \tau_{n}\right) B$ for each $n \in \mathbb{N}$, we see that $B$ is local with maximal ideal $\mathbf{n}=(x, y) B$. Since $B$ and $A$ are both dominated by $k[[x, y]]$, it follows that $A$ dominates $B$.

To prove that $U$ and $B$ are UFDs, we use that $U_{n}$ is a polynomial ring over a field and $U_{n}\left[\frac{1}{x y}\right]=U\left[\frac{1}{x y}\right]$. Hence the ring $U\left[\frac{1}{x y}\right]$ is a UFD. For each $n \in \mathbb{N}$, the principal ideals $x U_{n}$ and $y U_{n}$ are prime ideals in the polynomial ring $U_{n}$. Therefore $x U$ and $y U$ are principal prime ideals of $U$. Moreover, $U_{x U}=B_{x B}$ and $U_{y U}=B_{y B}$ are DVRs since each is the contraction to the field $k(x, y, \sigma, \tau)$ of the $(x)$-adic or the $(y)$-adic valuations of $k[[x, y]]$. A theorem of Nagata [9, Theorem 6.3, p. 21] implies that $U$ is a UFD; see also [4, Theorem 2.9 and Fact 2.11]. Since $B$ is a localization of $U$, the $\operatorname{ring} B$ is a UFD. This completes the proof of items 1 and 2 .

Since $B$ is dominated by $k[[x, y]]$, the intersection $\cap_{n=1}^{\infty} \mathbf{n}^{n}=(0)$, and so $B$ is Hausdorff in the topology defined by the powers of $\mathbf{n}$. We have local injective maps $R \hookrightarrow B \hookrightarrow \widehat{R}$, and for each positive integer $n$, we have $\mathbf{m}^{n} B=\mathbf{n}^{n}, \mathbf{m}^{n} \widehat{R}=\widehat{\mathbf{m}}^{n}$ and $\widehat{\mathbf{m}}^{n} \cap R=\mathbf{m}^{n}$. Since the natural map $R / \mathbf{m}^{n} \rightarrow \widehat{R} / \mathbf{m}^{n} \widehat{R}=\widehat{R} / \widehat{\mathbf{m}}^{n}$ is an isomorphism, the map $R / \mathbf{m}^{n} \rightarrow B / \mathbf{m}^{n} B=B / \mathbf{n}^{n}$ is injective and the map $B / \mathbf{n}^{n} \rightarrow$ 
$\widehat{R} / \mathbf{n}^{n} \widehat{R}=\widehat{R} / \widehat{\mathbf{m}}^{n}$ is surjective. Since $B / \mathbf{n}^{n}$ has finite length as an $R$-module, it follows that $R / \mathbf{m}^{n} \cong B / \mathbf{n}^{n} \cong \widehat{R} / \widehat{\mathbf{m}}^{n}$ for each $n \in \mathbb{N}$ and hence $\widehat{B}=\widehat{R}=k[[x, y]]$. Notice that $B$ is a birational extension of the three-dimensional Noetherian domain $C[y, \tau]$. The dimension of $B$ is at most 3 by a theorem of Cohen, [6, Theorem 15.5] or [4, Theorem 2.9]. This completes the proof of items 3,4 and 5 .

For item 6 , since $A$ is a two-dimensional regular local ring, $(a) \Longrightarrow(b)$. Clearly $(b) \Longrightarrow(c)$. Since $B$ is local by item 2 , and since the completion of a Noetherian local ring is a faithfully flat extension, we have $(c) \Longrightarrow(d)$. It is clear that $(d) \Longrightarrow(e)$. To complete the proof of Theorem 4.2, it suffices to show that $(e) \Longrightarrow(a)$. Since $A$ birationally dominates $B$, we have $B=A$ if and only if $b A \cap B=b B$ for every element $b \in \mathbf{n}$. The principal ideal $b B$ is closed in the $\mathbf{n}$-adic topology on $B$ if and only if $b B=b \widehat{B} \cap B$. Also $\widehat{B}=\widehat{A}$ and $b A=b \widehat{A} \cap A$, for every $b \in B$. Thus (e) implies, for every $b \in B$,

$$
b B=b \widehat{B} \cap B=b \widehat{A} \cap B=b \widehat{A} \cap A \cap B=b A \cap B,
$$

and so $B=A$. This completes the proof of Theorem 4.2

Depending on the choice of $\sigma$ and $\tau$, the $\operatorname{ring} B$ may fail to be Noetherian. Example 4.3 shows that in the setting of Theorem 4.2 the ring $B$ can be strictly smaller than $A:=k(x, y, \sigma, \tau) \cap k[[x, y]]$.

Example 4.3. Using the setting of Notation 4.1 let $\tau \in k[[y]]$ be defined to be $\sigma(y)$, that is, set $b_{i}:=a_{i}$ for every $i \in \mathbb{N}$. We then have that $\theta:=\frac{\sigma-\tau}{x-y} \in A$. Indeed,

$$
\sigma-\tau=a_{1}(x-y)+a_{2}\left(x^{2}-y^{2}\right)+\cdots+a_{n}\left(x^{n}-y^{n}\right)+\cdots,
$$

and so $\theta=\frac{\sigma-\tau}{x-y} \in k[[x, y]] \cap k(x, y, \sigma, \tau)=A$. As a specific example, one may take $k:=\mathbb{Q}$ and set $\sigma:=e^{x}-1$ and $\tau:=e^{y}-1$. The $\operatorname{ring} B$ is a localization of the ring $U:=\bigcup_{n \in \mathbb{N}} k\left[x, y, \sigma_{n}, \tau_{n}\right]$.

Claim 4.4. The element $\theta$ is not in $B$.

Proof. If $\theta$ is an element of $B$, then

$$
\sigma-\tau \in(x-y) B \cap U=(x-y) U .
$$

Let $S:=k[x, y, \sigma, \tau]$ and let $U_{n}:=k\left[x, y, \sigma_{n}, \tau_{n}\right]$ for each positive integer $n$. We have

$$
U=\bigcup_{n \in \mathbb{N}} U_{n} \subseteq S\left[\frac{1}{x y}\right] \subset S_{(x-y) S},
$$

where the last inclusion is because $x y \notin(x-y) S$. Thus $\theta \in B$ implies that

$$
\sigma-\tau \in(x-y) S_{(x-y) S} \cap S=(x-y) S,
$$

but this contradicts the fact that $x, y, \sigma, \tau$ are algebraically independent over $k$, and thus $S$ is a polynomial ring over $k$ in $x, y, \sigma, \tau$. 
Therefore $\frac{\sigma-\tau}{x-y} \notin B$, and so $B \subsetneq A$ and $(x-y) B \subsetneq(x-y) A \cap B$. Since an ideal of $B$ is closed in the $\mathbf{n}$-adic topology if and only if the ideal is contracted from $\widehat{B}$ and since $\widehat{B}=\widehat{A}$, the principal ideal $(x-y) B$ is not closed in the $\mathbf{n}$-adic topology on $B$. Using Theorem 4.2, we conclude that $B$ is a non-Noetherian three-dimensional local Krull domain having a two-generated maximal ideal such that $B$ birationally dominates a four-dimensional regular local domain. In this connection, also see [4, Example 8.11].

The setting of (4.1) is balanced in $x$ and $y$ in the sense that the roles of $x$ and $y$ are interchangeable. A more truly iterative process is described in Setting 4.5

Setting 4.5. Let $k$ be a field, let $x$ be an indeterminate over $k$, and let

$$
\sigma:=\sum_{i=1}^{\infty} a_{i} x^{i} \in k[[x]] \quad \text { with each } \quad a_{i} \in k
$$

be a formal power series that is algebraically independent over the field $k(x)$. As in Example 2.1, let $\sigma_{n}$ be the $n^{\text {th }}$ endpiece of $\sigma$ and define

$$
C_{n}:=k\left[x, \sigma_{n}\right]_{\left(x, \sigma_{n}\right)} \quad \text { and } \quad C:=k(x, \sigma) \cap k[[x]]=\underline{\lim }\left(C_{n}\right)=\bigcup_{n=1}^{\infty} C_{n} .
$$

Let $y$ be an indeterminate over $C$ and let

$$
\tau:=\sum_{i=1}^{\infty} b_{i} y^{i} \in C[[y]] \quad \text { with each } \quad b_{i} \in C
$$

be a formal power series that is algebraically independent over $C[y]$. Notice that as a special case we may have each $b_{i} \in k$. Let $\tau_{n}$ be the $n^{\text {th }}$ endpiece of $\tau$ and define

$$
\begin{aligned}
U_{n} & :=k\left[x, y, \sigma_{n}, \tau_{n}\right], \quad U:=\lim _{\longrightarrow} U_{n}=\bigcup_{n=1}^{\infty} U_{n} ; \\
B_{n} & :=k\left[x, y, \sigma_{n}, \tau_{n}\right]_{\left(x, y, \sigma_{n}, \tau_{n}\right)} \quad B:=\lim _{\longrightarrow}\left(B_{n}\right)=\bigcup_{n=1}^{\infty} B_{n} ; \\
D_{n} & :=C\left[y, \tau_{n}\right]_{\left(x, y, \tau_{n}\right)} \quad D:=\underline{\lim }\left(D_{n}\right)=\bigcup_{n=1}^{\infty} D_{n} ; \\
A & :=k(x, y, \sigma, \tau) \cap k[[x, y]] .
\end{aligned}
$$

Notice that each $U_{n} \subset k[[x, y]]$ and $U_{n}$ is a polynomial ring in $x, y, \sigma_{n}, \tau_{n}$ over the field $k$. Since $C \subset B$, we have $B=D$.

Remark 4.6. Let the notation be as in Setting 4.5. For certain choices of $\sigma$ and $\tau$, the ring $B$ is Noetherian with $B=A$. Let $k$ be the field $\mathbb{Q}$ of rational numbers. Thus $R:=\mathbb{Q}[x, y]_{(x, y)}$ is the localized polynomial ring in the variables $x$ and $y$, and the completion $\widehat{R}$ of $R$ with respect to its maximal ideal $\mathbf{m}:=(x, y) R$ is $\widehat{R}=\mathbb{Q}[[x, y]]$, the formal power series ring in $x$ and $y$. Let $\sigma:=e^{x}-1 \in \mathbb{Q}[[x]]$, 
and $C:=\mathbb{Q}[[x]] \cap \mathbb{Q}(x, \sigma)$. Thus $C$ is an excellent DVR with maximal ideal $x C$, and $T:=C[y]_{(x, y) C[y])}$ is an excellent countable two-dimensional regular local ring with maximal ideal $(x, y) T$ and with $(y)$-adic completion $C[[y]]$. The UFD $C[[y]]$ has maximal ideal $\mathbf{n}=(x, y)$. Since $T$ is countable, Theorem 3.8 implies that there exists $\tau \in C[[y]]$ that is primarily limit-intersecting in $y$ over $R$. Hence for this choice of $\sigma \in \mathbb{Q}[[x]]$ and $\tau \in C[[y]]$, we have $A=\mathbb{Q}(x, y, \sigma, \tau) \cap C[[y]]$ is Noetherian and equal to its approximation domain $D=B$.

To fit the setting of Notation 4.1 with $k=\mathbb{Q}$, one wants $\tau \in \mathbb{Q}[[y]]$ rather than $\tau \in C[[y]]$. An example with this more restrictive property is given in [4, Example 7.15].

\section{Weakly flat extensions that are not flat.}

Let $d$ be an integer with $d \geq 2$. We obtain in Theorem 4.7 extensions that satisfy $\mathrm{LF}_{d-1}$ but do not satisfy $\mathrm{LF}_{d}$; see Definition 2.713. Thus we obtain examples where the intersection domain $A$ is equal to its approximation domain $B$, but $A$ is not Noetherian.

Theorem 4.7. Let $(R, \mathbf{m})$ be a countable excellent normal local domain. Assume that $\operatorname{dim} R=d+1 \geq 3$, that $\left(x_{1}, \ldots, x_{d}, y\right) R$ is an $\mathbf{m}$-primary ideal, and that $R^{*}$ is the (y)-adic completion of $R$. Then there exists $f \in y R^{*}$ such that $f$ is algebraically independent over $R$ and the map $\varphi: R[f] \longrightarrow R^{*}[1 / y]$ is weakly flat but not flat. Indeed, $\varphi$ satisfies $L F_{d-1}$, but fails to satisfy $L F_{d}$. Thus the intersection domain $A:=\mathcal{Q}(R[f]) \cap R^{*}$ is equal to its approximation domain $B$, but $A$ is not Noetherian.

Proof. By Theorem 3.12, there exist elements $\tau_{1}, \ldots, \tau_{d} \in y R^{*}$ that are primarily limit-intersecting in $y$ over $R$. Let

$$
f:=x_{1} \tau_{1}+\cdots+x_{d} \tau_{d} .
$$

Using that $\tau_{1}, \ldots, \tau_{d}$ are algebraically independent over $R$, we regard $f$ as a polynomial in the polynomial ring $T:=R\left[\tau_{1}, \ldots, \tau_{d}\right]$. Let $S:=R[f]$. For $Q \in \operatorname{Spec} R^{*}[1 / y]$ and $P:=Q \cap T$, consider the composition $\varphi_{Q}$

$$
S \longrightarrow T_{P} \longrightarrow R^{*}[1 / y]_{Q}
$$

Since $\tau_{1}, \ldots, \tau_{d}$ are primarily limit-intersecting in $y$ over $R$, the map $T \hookrightarrow R^{*}[1 / y]$ is flat. Thus the map $\varphi_{Q}$ is flat if and only if the map $S \longrightarrow T_{P}$ is flat. Let $\mathbf{p}:=P \cap R$.

Assume that $P$ is a minimal prime of $\left(x_{1}, \ldots, x_{d}\right) T$. Then $\mathbf{p}$ is a minimal prime of $\left(x_{1}, \ldots, x_{d}\right) R$. Since $T$ is a polynomial ring over $R$, we have $P=\mathbf{p} T$ and $\operatorname{ht}(\mathbf{p})=d=\operatorname{ht} P$. Notice that $(\mathbf{p}, f) S=P \cap S$ and $\mathrm{ht}(\mathbf{p}, f) S=d+1$. Since a flat extension satisfies the going-down property, the map $S \longrightarrow T_{P}$ is not flat. Hence $\varphi$ does not satisfy $\mathrm{LF}_{d}$. 
Assume that ht $P \leq d-1$. Then $\left(x_{1}, \ldots, x_{d}\right) T$ is not contained in $P$. Hence $\left(x_{1}, \ldots, x_{d}\right) R$ is not contained in $\mathbf{p}$. Consider the sequence

$$
S=R[f] \hookrightarrow R_{\mathbf{p}}[f] \stackrel{\psi}{\longrightarrow} R_{\mathbf{p}}\left[\tau_{1}, \ldots, \tau_{d}\right] \hookrightarrow T_{P}
$$

where the first and last injections are localizations. Since the nonconstant coefficients of $f$ generate the unit ideal of $R_{\mathbf{p}}$, the map $\psi$ is flat; see [4, Theorem 11.20]. Thus $\varphi$ satisfies $\mathrm{LF}_{d-1}$.

We conclude that the intersection domain $A=R^{*} \cap \mathcal{Q}(R[f])$ is equal to its approximation domain $B$ and is not Noetherian.

We describe a specific example of Theorem 4.7

Example 4.8. Let $d$ be an integer with $d \geq 2$ and let $x_{1}, \ldots, x_{d}, y$ be indeterminates over a countable field $k$. Let $R$ be the localized polynomial ring in the variables $x_{1}, \ldots, x_{d}, y$ and let $R^{*}$ be the $(y)$-adic completion of $R$. Thus

$$
R=k\left[x_{1}, \ldots, x_{d}, y\right]_{\left(x_{1}, \ldots, x_{d}, y\right)} \quad \text { and } \quad R^{*}=k\left[x_{1}, \ldots, x_{d}\right]_{\left(x_{1}, \ldots, x_{d}\right)}[[y]] .
$$

As in Theorem 4.7, there exist elements $\tau_{1}, \ldots, \tau_{d} \in y R^{*}$ that are primarily limitintersecting in $y$ over $R$, and we consider $f:=x_{1} \tau_{1}+\cdots+x_{d} \tau_{d}$. By Theorem 4.7 the map $S \longrightarrow R^{*}[1 / y]$ satisfies $\mathrm{LF}_{d-1}$, but does not satisfy $\mathrm{LF}_{d}$. Thus the intersection domain $A=R^{*} \cap \mathcal{Q}(R[f])$ is equal to its approximation domain $B$ and is not Noetherian.

\section{REFERENCES}

[1] S. Abhyankar, Two notes on formal power series, Proc. Amer. Math. Soc. 7 (1956) 903-905.

[2] Y. Akizuki, Einige Bemerkungen über primäre Integritatsbereiche mit Teilerkettensatz, Proc. Phys-Math. Soc. Japan 17 (1935), 327-336.

[3] L. Burch, Codimension and analytic spread, Proc. Cambridge Philos. Soc. 72 (1972) 369-373.

[4] W. Heinzer, C. Rotthaus and S. Wiegand, Power series over Noetherian rings, book in progress.

[5] G. Leuschke and R. Wiegand, Cohen-Macaulay representations, Mathematical Surveys and Monographs, 181. American Mathematical Society, Providence, RI, 2012.

[6] H. Matsumura, Commutative ring theory, Cambridge Univ. Press, Cambridge, 1989.

[7] M. Nagata, An example of a normal local ring which is analytically reducible, Mem. Coll. Sci., Univ. Kyoto 31 (1958) 83-85.

[8] M. Nagata, Local Rings, John Wiley, New York, 1962.

[9] P. Samuel, Unique Factorization Domains, Tata Inst. of Fundamental Research, Bombay (1964).

[10] F.K. Schmidt, Über die Erhaltung der Kettensätze der Idealtheorie bei beliebigen endlichen Körpererweiterungen, Math. Z. 41 (1936), 443-450.

[11] R. Y. Sharp and P. Vamos, Baire's category theorem and prime avoidance in complete local rings, Arch. Math. 44 (1985) 243-248.

[12] P. Valabrega, On two-dimensional regular local rings and a lifting problem, Annali della Scuola Normale Superiore di Pisa 27 (1973) 1-21.

[13] R. Wiegand and S. Wiegand, The maximal ideal space of a Noetherian ring, J. Pure Appl. Algebra 8, (1976) 129-141. 
Department of Mathematics, Purdue University, West Lafayette, Indiana 47907

E-mail address: heinzer@math.purdue.edu

Department of Mathematics, Michigan State University, East Lansing, Mi 48824-1027

E-mail address: rotthaus@math.msu.edu

Department of Mathematics, University of Nebraska, Lincoln, NE 68588-0130

E-mail address: swiegand@math.unl.edu 